\title{
An investigation on the growth and characterization of DAST crystals grown by two zone growth technique
}

\author{
A.S. Haja Hameed ${ }^{\mathrm{a}}$, W.C. Yu ${ }^{\mathrm{b}, *}$, Z.B. Chen ${ }^{\mathrm{b}}$, C.Y. Tai ${ }^{\mathrm{a}}$, C.W. Lan ${ }^{\mathrm{a}}$ \\ ${ }^{a}$ Department of Chemical Engineering, National Taiwan University, Taipei, Taiwan 10617, ROC \\ ${ }^{\mathrm{b}}$ Department of Molecular Science and Engineering, National Taipei University of Technology, Taipei 106, Taiwan, ROC
}

Received 4 April 2005; received in revised form 25 April 2005; accepted 26 April 2005

Available online 20 June 2005

Communicated by K. Nakajima

\begin{abstract}
Two zone growth technique (TZGT) is reported for the growth of $4^{\prime}$-dimethylamino- $N$-methyl-4-stilbazolium tosylate (DAST) crystals, being one of the best organic materials for second-order nonlinear optics. The growth rates are estimated with respect to the temperature difference $(\Delta T)$ between the hot zone and cold zone. The grown crystals are morphologically interpreted. The crystal quality is examined from the XRD double crystal rocking curve. The microhardness number has been estimated for the DAST crystals grown at the different $\Delta T$ s. A good optical transmission is found in the cleaved plate of grown DAST crystal. The formation of microcrystals is observed on the (0 00 1) face.

(C) 2005 Elsevier B.V. All rights reserved.
\end{abstract}

PACS: 81.10.Dn; 47.27.Te

Keywords: A1. Convection; A1. Crystal morphology, X-ray diffraction; A2. Growth from solution; B1. Organic compounds; B2. Nonlinear optical materials

\section{Introduction}

Organic nonlinear optical (NLO) materials have been intensely investigated due to their high nonlinearities, high laser damage threshold, etc., compared to inorganic NLO materials. Among the technologically important crystals, DAST is one of

\footnotetext{
*Corresponding author. Tel./fax: + 886223633917.

E-mail address: wanchin@ntut.edu.tw (W.C. Yu).
}

the best crystals for both NLO and Electro-optic (EO) applications [1-5]. It offers extremely large electro-optic coefficient of $r_{111}=77 \pm 8 \mathrm{pm} / \mathrm{V}$ [6]. The material can also be used in terahertz frequency generation, telecommunications, etc. [7-11]. However its crystal growth still remains uneasy with sufficient quality and hardness from the application point of view. In fact, the slope nucleation method (SNM) could be a potential technique for the growth of high-quality DAST 
crystals $[12,13]$. Moreover, the number of nuclei is still difficult to control so that too many crystals stick together making them unsuitable in applications. Meanwhile, many small crystals appear at the bottom of the vessel, thereby reducing the growth yield notably. Even though the spontaneous nucleation method is good for high quality crystal growth, it is almost impossible to control the position of the crystal growth. Also, the crystal becomes poly crystals easily and sticks to the bottom of the vessel. The main limitation in the growth of large size organic crystals by the traditional techniques is growth rates of only $0.1-0.5 \mathrm{~mm} /$ day typical for low-temperature solution growth. Slow growth leads to growth cycles exceeding more months. The problems of equipment reliability, the high risk of failure, and defect formation during such long periods result in low yield and high cost of the final crystals. These reasons continue to stimulate the development of new techniques to accelerate the growth without sacrificing optical quality of large crystals [14].

Considering the above difficulties encountered during the DAST crystal growth, we established a new approach to grow DAST crystals by means of two-zone (TZ) growth, where the crystallization zone (cold zone) and dissolving zone (hot zone) were thermally separated. The DAST crystals were grown by this two zone growth technique (TZGT), where the solute is transported to the crystal through a combined effect of convection and diffusion. The growth rate with different hot and cold zone temperatures is discussed. In order to examine the quality of the grown crystals, the double X-ray diffraction results are presented. The grown crystals are characterized by microhardness tester, UV-VIS-NIR spectroscopy and optical microscopy.

\section{Experimental procedure}

\subsection{Synthesis}

DAST was synthesized by reacting 4-picoline and methyl toluenesulphonate at 1:1 molar ratio for $2 \mathrm{~h}$ and $30 \mathrm{~min}$, and treating the product (4-methyl- $N$-methyl pyridinium tosylate) with 4$N, N$-dimethylaminobenzaldehyde in equivalent molar ratio for $6 \mathrm{~h}$ using piperidine as a catalyst. The synthesis process was carried out inside a glove box with nitrogen atmosphere and the synthesized DAST material was further purified by recrystallization using methanol as solvent below $50{ }^{\circ} \mathrm{C}$. The twice-recrystallized materials from methanol were used for the crystal growth runs by TZGT.

\subsection{Crystal growth system}

Fig. 1 represents the schematic diagram of the crystal growth system. In this apparatus, a growth cell consisted of two zones viz., the upper zone was a cylindrical glass cell of capacity $400 \mathrm{ml}$ having a proper slope at its bottom and lower zone was a $100 \mathrm{ml}$ round bottom flask with a long neck. The cell and the flask are joined together through a channel of $1 \mathrm{~cm}$ in diameter, as shown in the diagram. The joined cylindrical cell and round

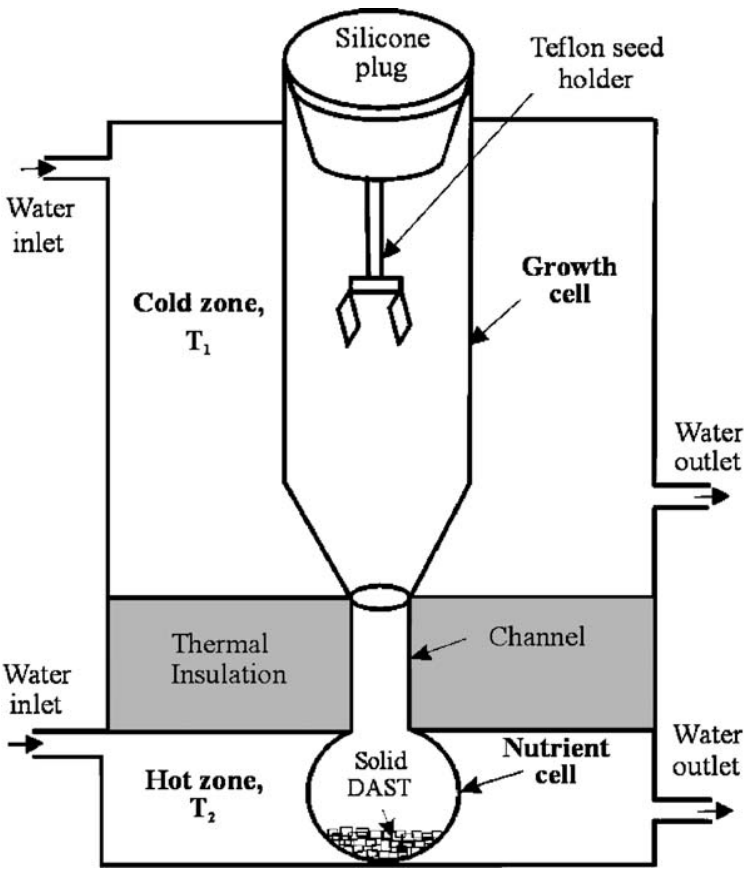

Fig. 1. Schematic drawing of the crystal growth system. Thermal insulation thickness: $4 \mathrm{~cm}$, cold zone cell height: $19 \mathrm{~cm}$, diameter: $6 \mathrm{~cm}$, and hot zone flask height: $7 \mathrm{~cm}$. 
bottom flask was the two-zone growth system for present investigation. The growth system was placed in a $50 \mathrm{~cm} \times 40 \mathrm{~cm} \times 28 \mathrm{~cm}$ acrylic container that was divided into the hot zone and the cold zone by a thermal insulating sheet. Water was continuously circulated through the cold and hot zones, respectively, from two constant temperature baths with a control accuracy of $\pm 0.01^{\circ} \mathrm{C}$.

One of the physical parameters involved in the SNM is the slope inclination angle, which is $30^{\circ}$ for optimal slope inclination. It is not advisable to make the angle steeper or smaller because of the possibility of the crystals to slip down from the slops even when they are tiny [12]. In our technique, the slope at the bottom of the growth cell plays an important role for the spontaneously nucleated tiny crystals to be slipped down. So, a considerable effect was taken to make the slope inclination angle, which is larger than the angle used in the SNM. The slope angle was made such that the tiny crystals if formed by spontaneous nucleation could slide down into the hot zone. Since the zone was hotter than the growth zone, the spontaneously nucleated crystals were dissolved there. As a result, only the seeded crystal grew in the cold zone.

\subsection{Growth rate measurements and characterizations}

Tiny DAST crystals obtained from spontaneous crystallization were poured to the hot zone using a glass tube. The growth cell was filled with the $400 \mathrm{ml}$ of DAST solution saturated at $40^{\circ} \mathrm{C}$ in methanol. Thus, excess solute was present at the bottom of the cell. The experiments were conducted for the different $\Delta T$ s viz., $3,5,7,10^{\circ} \mathrm{C}$. Time of applying the temperature difference to the saturated solution and inserting the seed crystal were the same for all the selected $\Delta T$ s. Also the cold zone solution was initially made to be slightly under saturation, which was done by warming the solution so that the suspended solute particles present in the solution was dissolved. Equal sized crystals of DAST obtained previously from methanol by spontaneous nucleation were used as seeds to start the growth runs. A typical seed holder was made of Teflon to avoid heterogeneous nucleation. Before the growth process, the saturated solution with the seeds was overheated by $0.5^{\circ} \mathrm{C}$. When the crystal edges were found to be dissolved, the solution temperature was brought down to the desired saturation temperature to initiate the growth. In this way, the rough boundary layer between the seed and grown crystals could be avoided. Then the temperatures of the hot zone $\left(T_{2}\right)$ and cold zone $\left(T_{1}\right)$ were set so as to give a desired $\Delta T$. The same procedure was adopted for different $\Delta T$ 's. The growing DAST crystal is shown in Fig. 2. In the figure, one of the edges of each crystal was pasted on the Teflon holder before starting the growth runs.

Seeds with a natural morphology were chosen for studying the growth rates along [0 0101$],\left[\begin{array}{lll}0 & 1 & 0\end{array}\right]$ and $\left[\begin{array}{lll}1 & 0 & 0\end{array}\right]$ at different $\Delta T$ levels. Prior to the experiments, the dimensions of the seeds were measured using an optical microscope with a micrometer scale. Seed crystals were inserted at a specified $\Delta T$. In the study, the $\Delta T$ was chosen as 3 , 5,7 and $10^{\circ} \mathrm{C}$. Consistent care has been taken about the time between applying the temperature gradient to the solution and inserting the seed crystal. At the end of the experiment, the crystal was harvested from the solution and an inert fluid, $3 \mathrm{M}$ Fluorinert electronic liquid FC-77, was used to remove the solvent adsorption on the crystal surface; FC-77 does not wet DAST crystals. Then the crystal dimensions were measured to compute the growth rates. The experiments were repeated several times on good quality seeds with no visible inclusions. The fluctuations in

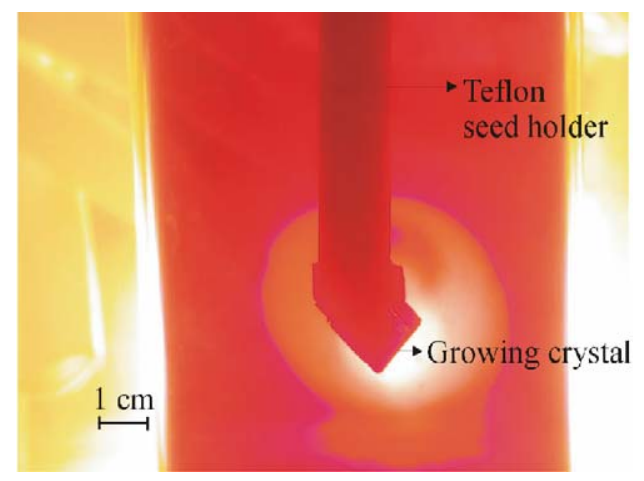

Fig. 2. Growing DAST crystal in the growth cell. 
the crystal growth rate in all the experiments fell in between $10 \%$ and $15 \%$.

The crystallinity was examined by analyzing the XRD double-crystal rocking curve (Model RUH3R, Rigaku, Japan) of the (l0 01 1) surface of the crystals, where FWHM was measured for the morphologically completed grown crystals. Moreover, Vickers hardness measurements were done on $\left(\begin{array}{ll}0 & 0\end{array}\right)$ face as well for the crystals grown at different $\Delta T$ s using a micro-hardness tester (Model HMV-2, Shimadzu, Japan). Using a spectrometer (Model: Jasco 570, Japan), the crystal transmission ranging from the visible to near-IR region was measured. A cleaved c-cut plate of $2 \mathrm{~mm}$ thickness was polished and then subjected to the above spectroscopic analysis. Optical microscope (Model: OLYMPUS BX51) was used to examine the surface feature of $\left(\begin{array}{lll}0 & 0 & \overline{1}\end{array}\right)$ of the DAST crystals.

\section{Results and discussion}

The usage of temperature gradients has been realized by many workers to create supersaturation in solutions [15]. The nucleation control with a considerably low nucleus formation is important in improving the growth method. Because the growth units have to be carried through the mobile mother phase to the growing crystal, the growth ability of a given crystal from the liquid phase usually increases with increasing equilibrium concentration of the mobile phase. Indeed, the main differences between the various methods are of technique rather than principle. It has long been known that diffusive mass transfer limitation can have a profound effect on solution crystal growth system. Such effects include unacceptably slow growth rates, unwanted modification of the crystal growth habit, spurious nucleation events and morphological instability.

In the present study, it is essential that there should be a constant flow of supply material into the growth zone, the quantities sufficient to provide for a specific rate of growth on the seed. The temperature of the experiments was varied from 40 to $55^{\circ} \mathrm{C}$. The supersaturation in the growth zone was varied by adopting different $\Delta T$ s.
In order to stream-up the solute from one regime to another, the growth system was designed to heat the lower dissolving section isothermally, which was hotter than the upper growth region which also maintained isothermal. The "channel" within the vessel separating the dissolving and growth regions aids in localizing the temperature gradient. In this method, an appropriate temperature gradient will produce a satisfactory supersaturation.

As discussed above, the hot saturated solution flew upwards to the cooler region of the cell maintained at $T_{1}$. So, supersaturation at the top of the cell was arrived by the solute transport through thermal convection and diffusion. Induced spontaneous nucleation occurred due to the sudden supercooling imposed by the upward-streaming of DAST solution. After nucleation, growth conditions were approaching constant since (i) both the temperature and concentration gradients were balanced by thermal convection and diffusion. So a constant supersaturation was around the growing seed and (ii) the growth temperature, i.e., cold zone temperature $T_{1}$ was kept constant. The $\Delta T$-induced growth proceeded through the solute transport. Here the temperature difference, $\Delta T$ was found proportional to the growth rate. As the growth rate was slow, crystal quality was improved, but the yield was reduced. In this study, a small $\Delta T$ was still preferred to ensure the crystal quality, but the growth rate was not too low. In this method, $\Delta T$ should be optimized for a fixed growth temperature.

For the present DAST crystal growth studies, the key variables that affected the rate were found to be: (1) the material purity and nutrient concentration; (2) crystallization temperature; (3) the temperature difference $\Delta T$ between the dissolving and crystallization zone, which in other words the concentration difference $\Delta C$; and (4) the seed orientation. When the temperature gradient was applied to the DAST solution, concentration gradient was not established until some time later. Then the cold zone concentration reached a sufficient supersaturation for growth to proceed. The growing crystal continued to absorb subsequent solute molecules, which diffused from the hot zone into the cold zone. Eventually, as the 
steady state was reached, the rate of growth equaled the rate of solute transport. As there was no further change in the concentration gradient once growth occurred, the rate remained constant.

Even though time of applying temperature difference was set to be the same, the arrival time of a steady state was found to be different for the different $\Delta T$ experiments, which was microscopically observed from the dissolution or growth on the seed crystal edges. For higher $\Delta T$, the time was shorter than that for lower $\Delta T$ due to high streaming-up of solute which is evidenced from the fact that higher growth rate was achieved at higher $\Delta T$. In addition, all the growth experiments were executed after the arrival of steady state so as to obtain a constant flow of supply material into the growth zone.

Due to the existence of convection, a mass transport was not sorely due to the diffusion. Nevertheless, the mass transfer rate, or the growth rate, could still be represented as a function of the temperature difference. In general, the mass transfer taking place during the crystallization of DAST varied about linearly with $\Delta T$. However, for a certain critical supersaturation $\Delta T_{\text {crr }}$ the mass transfer was found almost zero. It is also expected that natural convection and geometry of the growth system play important roles for the growth rate.

Fig. 3 shows DAST crystal grown for the period of 12 days at $\Delta T=7^{\circ} \mathrm{C}$ (Cold zone temperature is

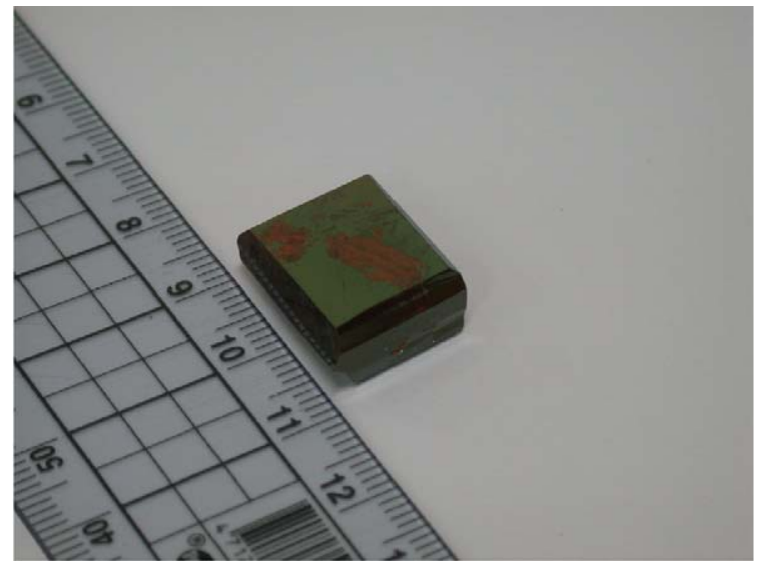

Fig. 3. DAST crystal grown for the period of 12 days at $\Delta T=$ $7{ }^{\circ} \mathrm{C}$ $45^{\circ} \mathrm{C}$ and hot zone is $52^{\circ} \mathrm{C}$ ). In case of the grown crystal (Fig. 3), under steady state, the difference between the actual $\Delta T=5^{\circ} \mathrm{C}$ (at the crystal growth position) and set $\Delta T=7{ }^{\circ} \mathrm{C}$ (before steady state) with respect to the hot zone temperature was about $2{ }^{\circ} \mathrm{C}$, which is due to convection and diffusion of the solution. The difference is also dependent on the diameter of the channel. In the grown crystal, the large $\left(\begin{array}{ll}0 & 0\end{array}\right)$ face was found and it was bounded by $\left\{\begin{array}{lll}1 & 1 & 0\end{array}\right\},\left\{\begin{array}{lll}1 & \overline{1} & 0\end{array}\right\}$ and small $\left\{\begin{array}{lll}0 & 2 & 0\end{array}\right\}$ faces where the $\{020\}$ faces were found to disappear in the prolonged growth in this technique. The DAST crystals tend to grow along the crystallographic $a$-axis under low supersaturation and along the $c$-axis under high supersaturation [16]. At higher $\Delta T$, high supersaturation was achieved as well as at lower $\Delta T$, low supersaturation was observed. For higher $\Delta T$, the growth rate along the $a$-axis was high so that there was bigger $\{110\}$ faces than the faces grown at lower supersaturation i.e., lower $\Delta T$. This result was consistent with the previous report [16]. The schematic morphological diagram of DAST crystals grown under high supersaturation is shown in Fig. 4(a). The DAST crystal grown at the supersaturation $\left(\Delta T=6{ }^{\circ} \mathrm{C}\right.$, cold-zone temperature $T_{1}=40^{\circ} \mathrm{C}$ ) is shown in Fig. 4(b). It is noted that the growth rate at $\left(\begin{array}{lll}0 & 0 & 1\end{array}\right)$ increases with the increasing growth temperature. Recently, DAST crystal was grown at $\Delta T=10^{\circ} \mathrm{C}$ with dimension of $32 \times 30 \times 8 \mathrm{~mm}^{3}$ with a growth rate of $1.7 \mathrm{~mm}$ (along $a$ and $b$ axes) per day in 17 days. Stirring

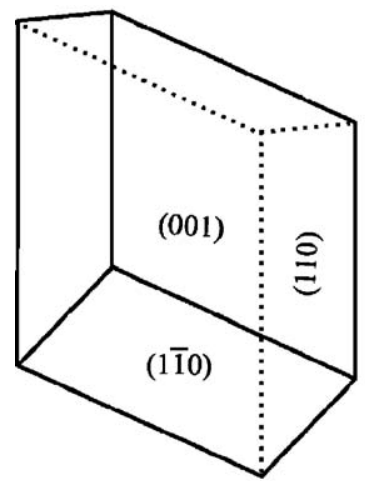

(a)

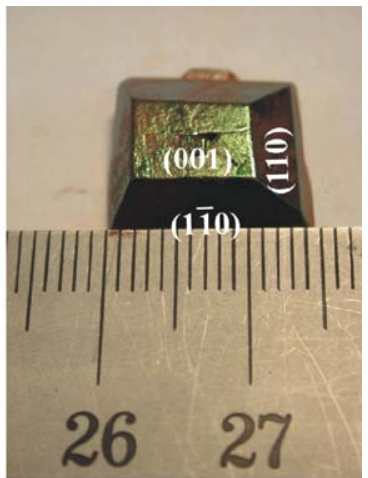

(b)
Fig. 4. (a) DAST crystal morphology at higher supersaturation and (b) grown DAST crystal at $\Delta T=6{ }^{\circ} \mathrm{C}$. 
was used at the hot zone $\left(55^{\circ} \mathrm{C}\right)$ with the cold zone temperature of $45^{\circ} \mathrm{C}$. Nevertheless, with such a high growth rate, the crystal quality was not good.

Under the same temperature gradient, the growth rates at different planes of the growing crystals were not the same. The growth rates $(R)$ measured along [0 001$]$, [ [ $\left.\begin{array}{lll}0 & 1 & 0\end{array}\right]$ and [ $\left[\begin{array}{lll}1 & 0 & 0\end{array}\right]$ with respect to $\Delta T$ are plotted in Fig. 5. From the figure, it was observed that the growth rate was roughly proportional to the temperature difference, $\Delta T$. The growth along [ $\left.\begin{array}{lll}1 & 0 & 0\end{array}\right]$ and $\left[\begin{array}{lll}0 & 1 & 0\end{array}\right]$ of the grown crystal was almost same. In all the crystals, the growth rate normal to $\left(\begin{array}{ll}0 & 0\end{array}\right)$ face was much slower as compared to that of $(100)$ and (0 10 l 0 ) faces.

In order to characterize the grown DAST crystals in terms of crystallinity, the double crystal $X R D$ rocking curve was recorded from which the FWHM was measured. The XRD rocking curve for the DAST crystal is shown in Fig. 6. The crystal grown at lower $\Delta T$, i.e., $7^{\circ} \mathrm{C}$, was used in this study. As discussed by Mori et al. [12], the homogeneity of molecular orientation mainly depends on the growth rate. The measured FWHM of the crystal was 576 arcsec, which was comparable to the FWHM (475 arcsec) of the DAST crystals grown without EDTA reported earlier [17]. However, the grown crystals in the previous reports were much smaller and were grown by a different method, by which it is not

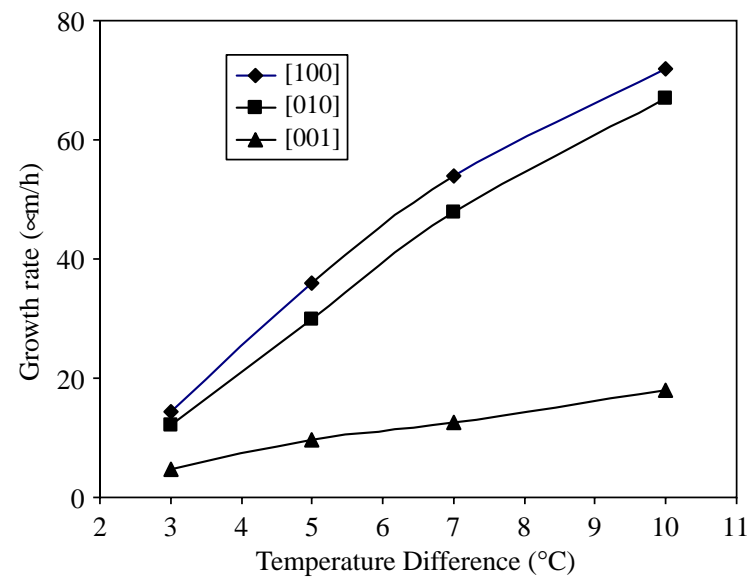

Fig. 5. Crystal growth rate $R$ measured as a function of temperature difference.

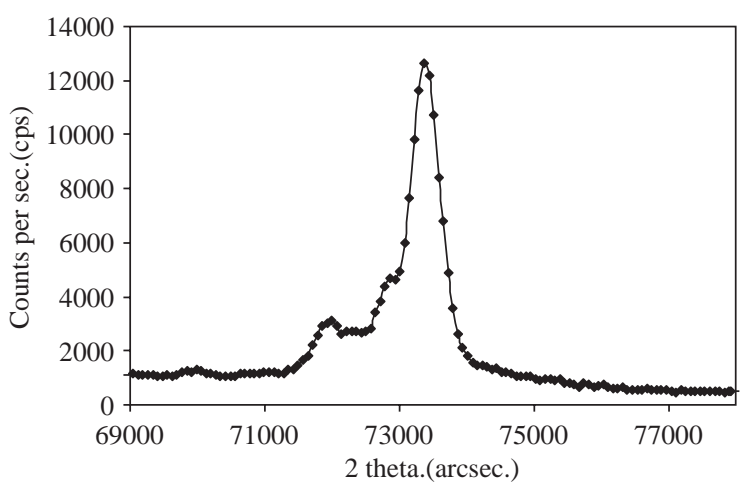

Fig. 6. XRD rocking curve for the DAST crystal.

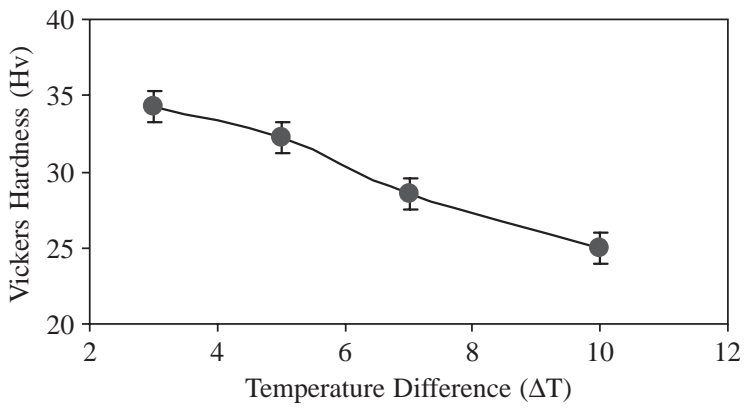

Fig. 7. Plot of microhardness number vs. temperature difference.

easy to get a large crystal. The broader FWHM of the present case reflected that the intrinsic crystal order is relatively lower which might be due to higher nutrient diffusion and higher growth rate.

The microhardness numbers were calculated for the DAST crystals grown at various $\Delta T$ s. Fig. 7 shows the plot of the hardness number vs. the temperature differences, for which the DAST crystals were grown. From the plot, it was observed that the microhardness decreased with the increase of the temperature difference $\Delta T$. The decrease in hardness was attributed from the imperfections due to solution inclusion and growth striations at higher supersaturation. The microhardness number has been found in the range $25-35 \mathrm{~kg} / \mathrm{mm}^{2}$ for the grown DAST crystals. For higher $\Delta T$, higher supersaturation was established in the cold zone due to the increased convection rate. The optical transmission spectrum of DAST 


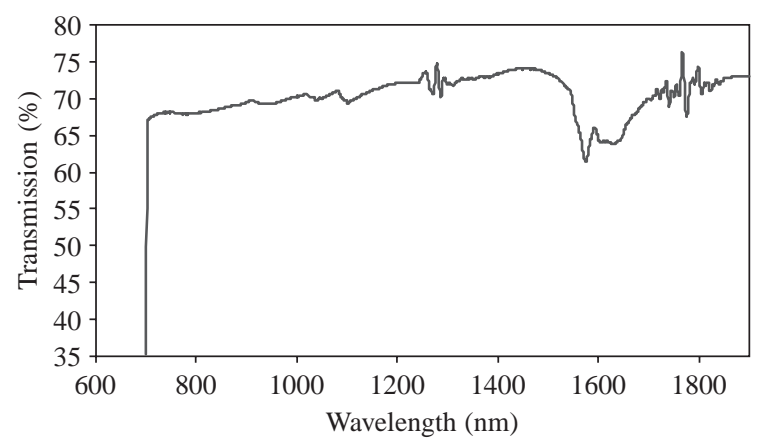

Fig. 8. Transmission spectrum of cleaved plate of DAST crystal. The thickness of the crystal is $2 \mathrm{~mm}$.

crystal is shown in Fig. 8. From the UV-VIS-NIR spectrum, it was found that the DAST crystal had about $70 \%$ of transmission. The transmission edge was observed at about $700 \mathrm{~nm}$, which was $100 \mathrm{~nm}$ right shift with the previous report [17]. The change in the transmission edge is due to the orientation of the cationic chromophore in the DAST crystal lattice with respect to its dielectric axes.

In the grown DAST crystals, the $\left(\begin{array}{lll}0 & 0 & \overline{1}\end{array}\right)$ plane of a microcrystal appeared preferentially on the $\left(\begin{array}{lll}0 & 0 & \overline{1}\end{array}\right)$ face. Thus, it was believed that the appearance of the $\left(\begin{array}{lll}0 & \overline{1}\end{array}\right)$ plane incorporated with the cleavage mechanism of the crystal. The large surface of the plate as $\left(\begin{array}{lll}0 & 0 & 1\end{array}\right)$ bounded by $\left\{\begin{array}{lll}1 & 1 & 0\end{array}\right\}$, $\left\{\begin{array}{lll}1 & 1 & 0\end{array}\right\}$ and $\left\{\begin{array}{lll}0 & 2 & 0\end{array}\right\}$ faces was observed in the grown DAST crystals. The $\{001\}$ planes of DAST microcrystals appeared on the back surface of DAST crystals. The morphology of the microcrystal is shown in Fig. 9a. The two dimensional view of the microcrystals is shown in Fig. 9b. It has microscopic $\left\{\begin{array}{lll}0 & 2 & 0\end{array}\right\},\left\{\begin{array}{lll}0 & 0 & \overline{1}\end{array}\right\}$ faces viewed perpendicular to $(00 \overline{1})$ face of the DAST crystal. The microcrystals are formed parallel to the $\langle 001\rangle$ direction, i.e., parallel to the cleavage plane $\left(\begin{array}{lll}0 & 0 & 1\end{array}\right)$. The microcrystals appeared only on the $\left(\begin{array}{lll}0 & 0 & \overline{1}\end{array}\right)$ face so that the roughness of the face was accomplished. The formation of microcrystals was not observed on the $(001)$ face leading to the smooth surface. The formation of microcrystals occurred at high supersaturation due to high $\Delta T$. The microcrystals are oriented in a regular pattern and did not touch with the tail edges of the successive

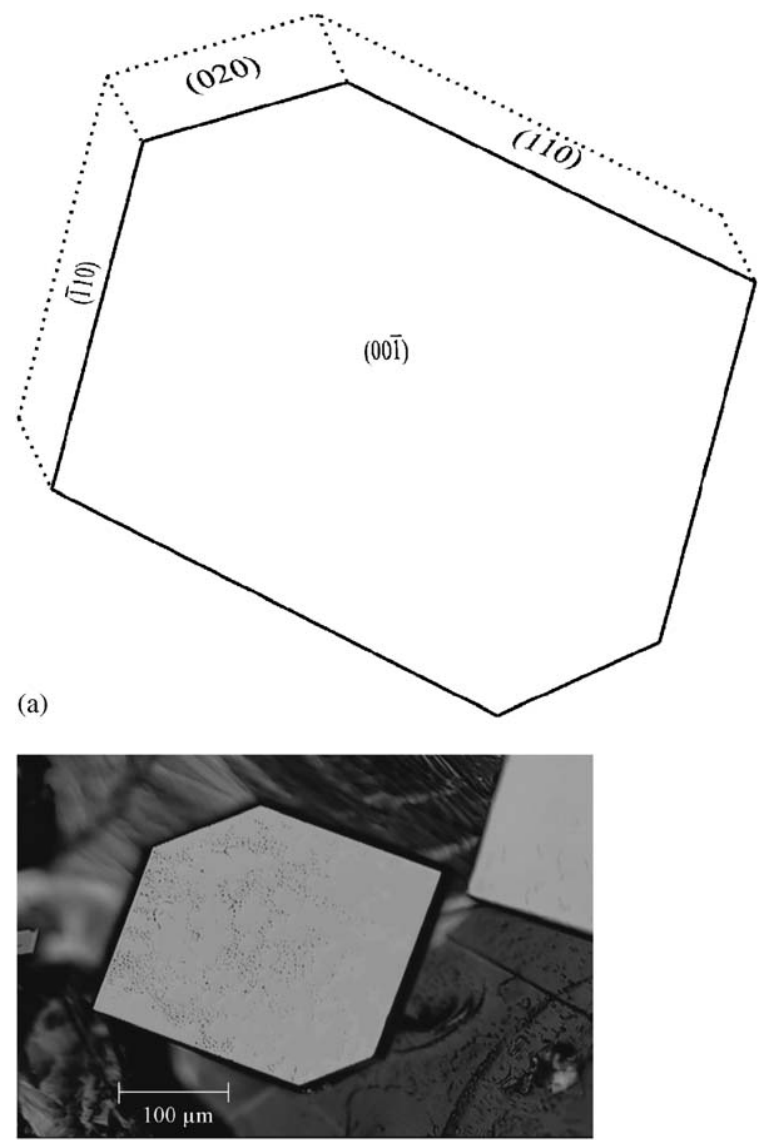

(b)

Fig. 9. (a) Morphology of two dimensional DAST microcrystal (dashed lines are not present in (b)) and (b) photograph of typical microcrystals observed on the $(00 \overline{1})$ face.

neighbors. In the bulk DAST crystals, the face (0 20$)$ was mostly disappeared after the prolonged growth whereas in the microcrystal, the boundary of $\left\{\begin{array}{lll}0 & 2 & 0\end{array}\right\}$ and $\left\{\begin{array}{lll}0 & \overline{1}\end{array}\right\}$ faces was clearly seen due to its perfect alignment parallel to the cleavage plane.

\section{Conclusion}

The two zone growth technique was reported to grow DAST crystals for the first time. The mechanism of the method, the crystal growth of DAST, and the growth rate with various temperature gradients were discussed. The growth rate of 
the crystal could be increased by varying the channel diameter and optimizing the temperature difference $\Delta T$. Suitable cell geometry would result in the size and quality of the grown crystal. By this technique, in case the crystals to be nucleated spontaneously in the growth zone could be allowed to slip down to the hot zone, where the crystals were dissolved. So the spontaneous nucleation problems were solved during the growth. Also its advantages lie in the simplicity of the available equipment, and a wide range of crystals could be grown with a large size from a solution having a narrow metastable zone width. From the microhardness studies, it was observed that the microhardness decreased with the increase of the temperature difference $\Delta T$. This was consistent with the previous studies that the crystal quality degraded with the increasing growth speed. A good optical transparency was observed in the cleaved plate of the crystal grown at small supersaturation. Nevertheless, the crystallinity of the grown DAST crystals was found not consistent with that of DAST crystals grown by the slope nucleation method. This was because of the more roughness of $\left(\begin{array}{lll}0 & 0 & \overline{1}\end{array}\right)$ face in the large crystals. It was found that

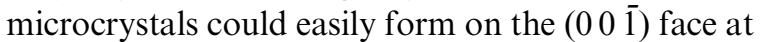
higher supersaturation in the growth zone. Apart from the above discussion, natural convection, the geometry configuration, size of the growth system, orientation to gravitation direction, etc. will play important roles. The results of the fluid flow during crystal growth and its theoretical understanding will be communicated in due course.

\section{References}

[1] H. Nakanishi, H. Matsuda, S. Okada, M. Kato, Proc. MRS. Int. Mtg. Adv. Mater. 1 (1989) 97.

[2] S.R. Marder, J.W. Perry, W.P. Schaefer, Science 245 (1989) 626.

[3] S.R. Marder, J.W. Perry, C.P. Yakymyshyn, Chem Mater. 6 (1994) 1137.

[4] R.W. Munn, C.N. Ironside, Principles and Applications of Nonlinear Optical, Materials, Chapman \& Hall, London, UK, 1993.

[5] P. Gunter, Non-linear Optical Effects and Materials, Springer, Berlin, Germany, 2000.

[6] F. Pan, G. Knofle, Ch. Bosshard, S. Follonier, R. Spreiter, M.S. Wong, P. Gunder, Appl. Phys. Lett. 69 (1996) 13.

[7] N.A.V. Dantzig, C.M. Planken, B.J. Huib, Opt. Lett. 23 (1998) 466.

[8] T.J. Carrig, G. Rodriguer, T.S. Clement, A.J. Taylor, K.R. Stewart, Appl. Phys. Lett. 66 (1995) 121.

[9] U. Meier, M. Bosch, Ch. Bosshard, F. Pan, P. Gunter, J. Appl. Phys. 83 (1998) 3486.

[10] U. Meier, M. Bosch, Ch. Bosshard, P. Gunter, Synth. Met. 109 (2000) 19.

[11] X.-C. Zhang, X.F. Ma, Y. Jin, T.-M. Lu, E.P. Boden, P.D. Phelps, K.R. Stewart, C.P. Yakymyshyn, Appl. Phys. Lett. 61 (1992) 3080.

[12] Y. Mori, Y. Takahashi, T. Iwai, M. Yoshimura, Y.K. Yap, T. Sasaki, Jpn. J. Appl. Phys. 39 (2000) L1006.

[13] Y. Takahashi, J. Yabuzaki, H. Adachi, Y. Mori, T. Sasaki, Nonlinear Opt. 22 (1999) 267.

[14] N.P. Zaitseva, J.J. De Yoreo, M.R. Dehaven, R.L. Vital, K.E. Montgomery, M. Richardson, L.J. Atherton, J. Crystal Growth 180 (1997) 255.

[15] R.A. Laudise, The Growth of Single Crystals, Prentice Hall, Englewood Cliffs, N.J., 1970.

[16] S. Sohma, H. Takahashi, T. Taniuchi, H. Ito, Chem. Phys. 245 (1999) 359.

[17] J.I. Wu, R. Gopalakrishnan, C.I.D. Tai, C.W. Lan, Jpn. J. Appl. Phys. 43 (2004) 1507. 